\title{
Process optimization of melt spinning and mechanical strength enhancement of functionalized multi-walled carbon nanotubes reinforcing polyethylene fibers.
}

\begin{abstract}
Carboxylic functional groups were introduced on multi-walled carbon nanotubes (MWCNTs) using the Ultraviolet (UV) Ozone treatment. Three melt spinning process parameters (spinning temperature, spinning distance, and the number of spinning revolutions) were evaluated by robust design (Taguchi's method) for a composite of fibers with the objective of enhancing the mechanical strength. The optimized melt spinning parameters were obtained. The predicted strength value of CNTs-PE fibers was determined using statistical analysis, and this value is close to the verification experiment value. Thus, robust design was successfully applied in this study. The crystallization of bulk pure PE was significantly increased by the formation of fibers through mechanical drawing of the melt spinning. The addition of CNTs in the polymer matrix accelerates the nucleation and crystal growth of the polymer. No CNT alignment in the PE matrix was observed on the sectioned surface of the fiber using Scanning Electron Microscopy (SEM). The degree of crystallization of the PE polymer plays an important role in the mechanical strength enhancement.
\end{abstract}

Keyword: Polymer (textile) fiber; B. Mechanical properties; C. Mechanical testing; E. Melt spinning. 